\title{
Axisymmetric photonic structures with PT-symmetry
}

\author{
Waqas W. Ahmed ${ }^{\mathrm{a}}$, Ramon Herrero ${ }^{\mathrm{a}}$, Muriel Botey ${ }^{\mathrm{a}}$, and Kestutis Staliunas ${ }^{\mathrm{a}, \mathrm{b}}$ \\ ${ }^{a}$ Departament de Física i Enginyeria Nuclear, Universitat Politècnica de Catalunya, Barcelona, Spain, ${ }^{\mathrm{b}}$ Institució \\ Catalana de Recerca i Estudis Avançats (ICREA), Barcelona, Spain.
}

\begin{abstract}
PT-symmetric structures in photonic crystals, combining refractive index and gain-loss modulations is becoming a research field with increasing interest due to the light directionality induced by these particular potentials. Here, we consider PT-symmetric potentials with axial symmetry to direct light to the crystal central point obtaining a localization effect. The axial and PT-symmetric potential intrinsically generates an exceptional central point in the photonic crystal by the merge of both symmetries. This particular point in the crystal lattice causes field amplitude gradients with exponential slopes around the crystal center. The field localization strongly depends on the phase of the central point and on the complex amplitude of the PT-potential.

The presented work analyzes in a first stage 1D linear PT-axisymmetric crystals and the role of the central point phase that determines the defect character, i.e. refractive index defect, gain-loss defect or a combination of both. The interplay of the directional light effect induced by the PT-symmetry and the light localization around the central point through the axial symmetry enhances localization and allows higher field concentration for certain phases. The linearity of the studied crystals introduces an exponential growth of the field that mainly depends on the complex amplitude of the potential. The work is completed by the analysis of 2D PT-axisymmetric potentials showing different spatial slopes and growth rates caused by symmetry reasons.
\end{abstract}

Keywords: PT-symmetry, Axisymmetric photonic structures, field localization

\section{INTRODUCTION}

Dispersion of light beams, including diffraction but also light diffusion, is a phenomenon limiting the performance of many linear and nonlinear optical devices ${ }^{1-6}$. Actually, in real materials diffusion is not independent to diffraction due to the non-instantaneous and causal response of the medium, as described by the Kramers-Kroning relations.

Artificial structured materials presenting spatial modulations of the refraction index and the gain or losses on the wavelength scale have demonstrated the ability to tailor light dispersion. For instance, a weak index modulation can reduce or totally elude diffraction while in-phase gain-loss and index modulations allow to tailor both, diffraction and diffusion ${ }^{7}$. One of the interesting symmetries for these complex spatial modulated materials is the PT-symmetry, where the real (imaginary) part of the modulation shows even (odd) symmetry. First introduced as a curiosity in quantum mechanics ${ }^{5}$, optical PT-symmetric systems have recently found realizations in optics attracting interest for holding new intriguing properties and novel unusual effects, especially when the PT-symmetry is broken. In the case of optics, the PT-symmetry condition requires that the complex refractive index, representing the actual refractive index and the gainloss distribution, fulfils: $n(r)=n^{*}(-r)$. For instance, this is fulfilled by any periodic index and gain-loss modulations dephased a quarter of the spatial period. As a matter of fact, the well-known Photonic Crystals (PhCs), with purely real modulations of the refractive index satisfy PT-symmetry. On the other extreme, systems with a constant real refractive index, holding only Gain-Loss Modulation (GLM) are also PT-symmetric. However in both cases the systems is perfectly symmetric. The most interesting situation arises when the real and gain-loss modulations are balanced, and the symmetric coupling between internal modes is broken. The system becomes strongly unidirectional leading to new features on light waves such as asymmetric unidirectional reflectors, invisible materials or input-dependent chirality in 2D systems ${ }^{8-14}$.

In order to understand the asymmetric new properties induced by the PT-symmetry, we first consider a Photonic Crystal in one-dimension (1D) with the simplest harmonic modulation that may be described by: $n(x)=n_{\operatorname{Re}} \cos (q x)$, being $q$ the spatial period of the index modulation. In turn, the complex refractive index of the simplest GLM may be analogously expressed by: $n(x)=n_{\mathrm{Re}}+i n_{\mathrm{Im}} \cos (q x)$. The PT-symmetry and its new peculiar properties arise when they are combined in a simultaneous modulation of dephased index and gain-loss modulations : $n(x)=n_{\operatorname{Re}} \cos (q x)+i n_{\operatorname{Im}} \sin (q x)$. The 
amplitudes of both modulations and particularly its ratio introduce the character of the complex modulated structure. At the PT-symmetry breaking point, when the amplitudes of both modulations are equal, $n_{\mathrm{Re}}=n_{\mathrm{Im}}$, the complex refractive index can be written as: $n(x)=n$ exp (iqx) and the resonant propagation modes, exp (ikx) and exp (-ikx), with $q=2 k$, become unidirectionally coupled, leading to the new asymmetric properties.

This work presents a new class of optical materials presenting and axis and PT-symmetric complex index modulations that enhance couplings between harmonics to the axis direction. This allows the concentration and enhancement of the optical field around axis.

\section{1D AXISYMMETRIC PT-SYMMETRY}

We start with the simple unidimensional case with axis located at $\mathrm{x}=0$ and a axi-symmetric potential represented in Fig.1. The complex refractive index modulation of Fig.1a presents a simple 1D harmonic modulation with the real and imaginary parts dephased by $\pm \pi / 2$ and yielding to a unidirectional coupling with opposite directions for $x<0$ and $x>0$.

The 1D harmonic optical refractive index represented in Fig.1 can be expressed as:

$$
n(x)=n_{\mathrm{Re}} \operatorname{Cos}(|x|+\phi)-i n_{\mathrm{Im}} \operatorname{Sin}(|x|+\phi)
$$

where, for simplicity, we use normalized spatial coordinates; $\mathrm{n}_{\mathrm{Re}}$ denotes the normalized amplitude of real component of the complex refractive index, and $\mathrm{n}_{\mathrm{Im}}$ the normalized amplitude of the imaginary component. The ratio between both amplitudes is expected to confer the asymmetric character to the system. Besides, the phase $\phi$, characterizes the potential at the symmetry centre, i.e. $\mathrm{x}=0$ and it is a crucial parameter for the field localization and enhancement, determining with the amplitudes signs the sense of the asymmetric mode coupling.
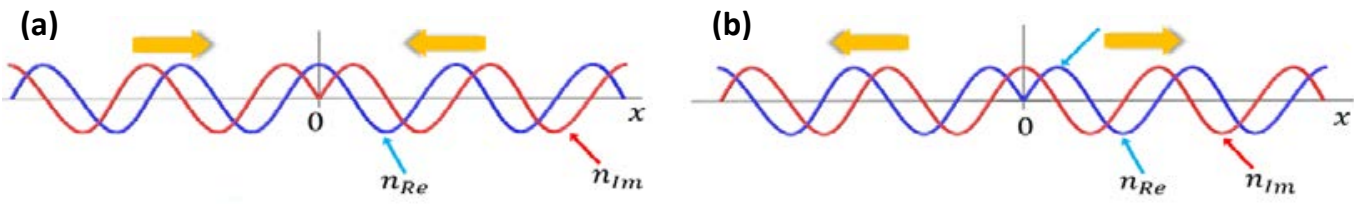

Figure 1. Local unidirectional coupling for the1D complex optical potential, where $n_{R e}$ in blue is the real index modulation, and $n_{I m}$ in red is the gain-loss modulation. In (a) the gain-loss modulation is positively dephased, the unidirectional coupling is directed inwards and a field concentration may be expected at $x=0$. In (b) the gain-loss modulation is negatively dephased and the unidirectional coupling is directed outwards.

In order to determine the propagation of a light beam in such structure, we consider the general paraxial electromagnetic field equation as a mathematical model, including diffraction and the potential, as:

$$
\partial_{t} A(x, t)=i \partial_{x}^{2} A(x, t)+i n(x) A(x, t)
$$

where $A(x, t)$ is the slowly varying amplitude envelope of the complex electromagnetic field. The three important parameters determining the spatial and temporal dynamics of such systems described by eq. 2 , are therefore $n_{\text {Re }}$, $n_{\text {Im }}$ and $\phi$. This system is analogous to the paraxial propagation of beams in a bidimensional structure with the same PTsymmetric complex index in the transverse direction $x$ and homogeneous along the longitudinal direction $z$ that takes the role of time.

Depending on the phase shift between gain-loss and index distributions the coupling is enhanced towards or from the central axis $x=0$. To intuitively deduce the light flow and field accumulation consider the field generation in positive gain areas and the light tendency to high refractive index zones.

We numerically solve eq.(2) with the complex refractive index of (1) by integrating the paraxial wave equation using the split step method, for a Gaussian initial beam of the form: $A(x)=\exp \left(-(x / w)^{2}\right)$ where the width of the initial beam profile $w$ should be large enough to obtain the modulation effect. Such a system may develop an extreme localization at $x=0$, exhibiting an exponential temporal growth due to the system linearity. We initially explore the parameter space $\left(n_{\mathrm{Re}}, n_{\mathrm{Im}}\right)$ 
for a fixed phase $\phi=0$. The envelope of the final field, after a sufficient propagation time, may be expressed as: $A(x) \square$ $\mathrm{e}^{\mathrm{i} \square t} \mathrm{e}^{\mathrm{i} k|x|}$ where $k=k_{R e}+\mathrm{i} k_{I m}$ and $\square=\square_{R e}+\mathrm{i} \square_{I m}$ are both complex functions. Note that while $k_{R e}$ is the spatial dominant mode $k_{I m}$ denotes the spatial localization exponent. Analogously, $\square_{I m}$ and $\square_{R e}$ stand for the temporal growth exponent and temporal oscillation frequency, respectively. Therefore, we obtain the localization exponent $k_{I m}$ (which we shall refer as localization) after a sufficient long time evolution, $t$, and the temporal exponential growth at $x=0, \square_{\text {Im }}$ (referred as growth throughout the text) as:

$$
k_{\mathrm{Im}}=\frac{d[\log (A(x, t))]}{d x}, \omega_{\mathrm{Im}}=\frac{d[\log (A(0, t))]}{d t}
$$

Such two main quantities fully characterize the field dynamics in the complex system, and are therefore used to identify the field localization and enhancement regimes in the parameter space $\left(n_{\text {Re }}, n_{\text {Im }}\right)$. Such results, for $\phi=0$, are presented on

Fig. 2.
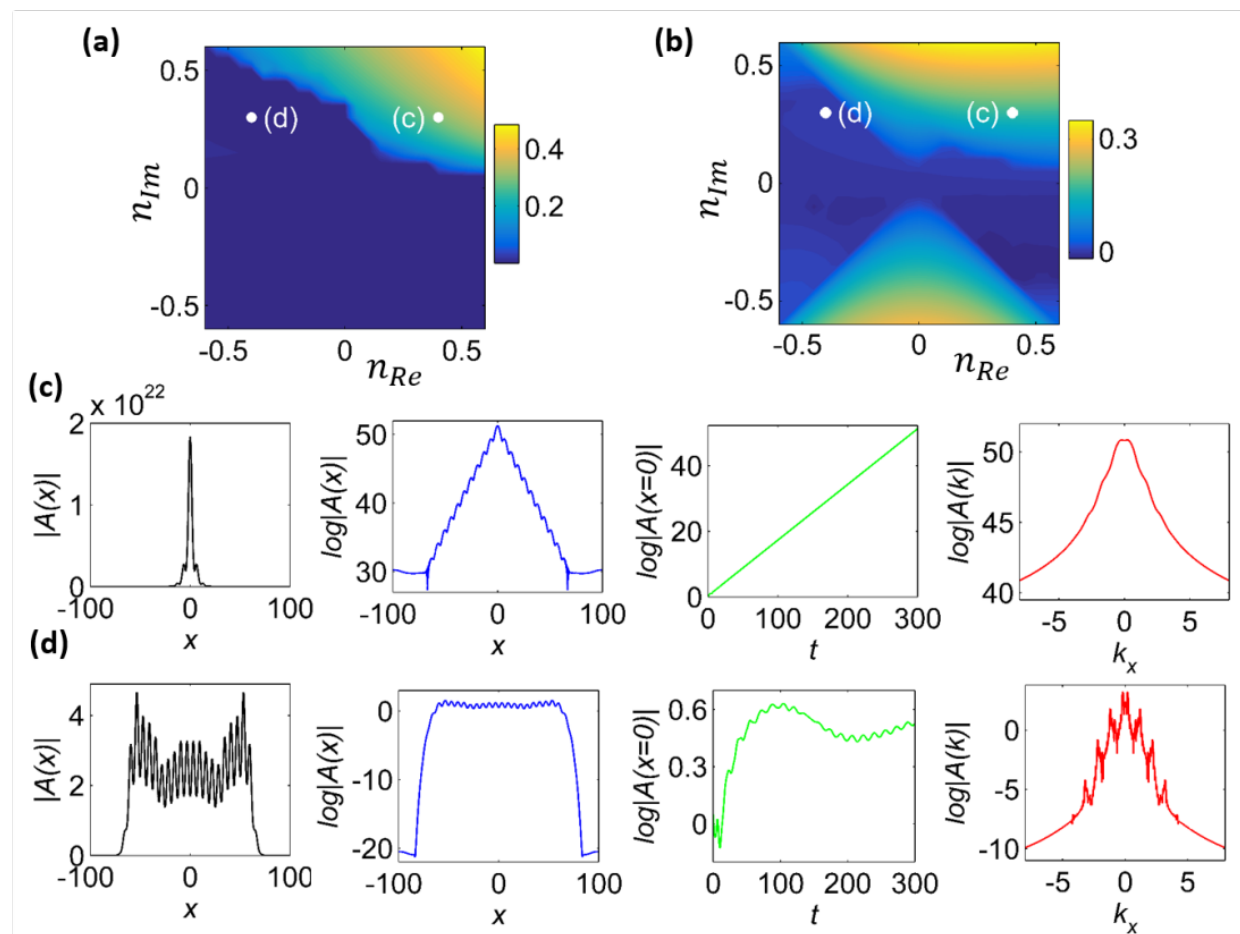

Figure 2. (a) Localization map, calculated after a sufficient long time (tD300 units) in the parameter space ( $\left.n_{\mathrm{Re}}, n_{\mathrm{Im}}\right)$ for $\phi=0$. (b) Growth map at the canter $(x=0)$, in the parameter space $\left(n_{\mathrm{Re}}, n_{\mathrm{Im}}\right)$. Two representative points marked as $(c),(d)$ correspond to the parameter sets $(0.4,0.3)$, and $(-0.4,0.3)$, respectively. In both cases, the spatial field profiles in real and logarithmic scale are shown on the first and second columns of (c) and (d) while the third and forth columns represent the temporal evolution of the field at the centre and the corresponding spatial field spectrum

The localization and growth maps built in parameter space are shown in Fig. 2a and Fig. 2b, respectively. We clearly observe in Fig. 2b a large growth for points fulfilling $\left|n_{I m}\right|>\left|n_{R e}\right|$ that denote an amplitude of the gain-loss modulation larger than the index one. The crossed lines given by $\left|n_{I m}\right|=\left|n_{R e}\right|$ correspond to the PT-symmetry breaking points. The growth profile seems to be not affected by the PT-symmetry breaking in quadrant $n_{I m}>0, n_{R e}>0$ denoting another effect associated to the phase $\phi$ that characterizes the potential at the symmetry centre. Thus, the largest growth occurs at the top right corner, coinciding with the simultaneous field enhancement on the same area of the parameter space, i.e. on the top right corner of both maps. To illustrate the effect of the PT-potential on the spatio-temporal field dynamics, we consider two representative points, within and outside the field localization regime, (c) and (d), having the same gain/loss 
modulation amplitude but opposite sign in the index amplitude modulation. While for the potential leads to an inward coupling of the wavevectors in point (c), the situation is reversed in point (d) where the coupling is outwards. The spatial field profile at (c) evidences a sharp localization of the field with an enhancement factor of $10^{22}$ at the canter while at (d) the field is spread. The field representation in logarithmic scale shows the expected exponential field localization in (c) and a flat field distribution in (d). Also the temporal evolution of field is exponentially growing in (c), but becomes flat after a short transient in (d). Both spatial field spectra are also provided, being its interpretation in (c) not obvious due to the strong exponential decay.

Following, we study the localization dependence on phase $\phi$ that characterizes the complex index in the central point $\mathrm{x}=0$. It is found that the highest localization regime is strongly phase dependent, being shifted in parameter space as it is summarized on Fig. 3. The localization maps for four representative phases: $\pi / 4, \pi / 2, \pi$ and $23 \pi / 16$ are presented in Figs 3a, 3b, 3c and 3d, where we observe how the localization rotates counterclockwise in parameter space. We find the amplitude values $n_{\mathrm{Re}}, n_{\mathrm{Im}}$ corresponding to the maximal localization exponent for each phase $\phi$ value. On Fig. 3e the maximum localization exponent is depicted as a function of $\phi$. Comparing these figures with Fig. 2a we see that the strongest field concentration is however achieved for $\phi=0$ and $\phi=\pi$ precisely when the coupling is inwards. The spatial potential profiles corresponding to the four phases are illustrated in Fig. 3e; note, for example, that while localization occurs for $n_{\mathrm{Re}}>0 n_{\mathrm{Im}}>0$ for $\phi=0$, it shifts to $n_{\mathrm{Re}}<0 n_{\mathrm{Im}}<0$ for $\phi=\pi$. Finally, we find that for very small gain still a minor localization is found, for high index contrast, see Fig. 3b and 3d, in cases where the coupling is not inwards but outwards.
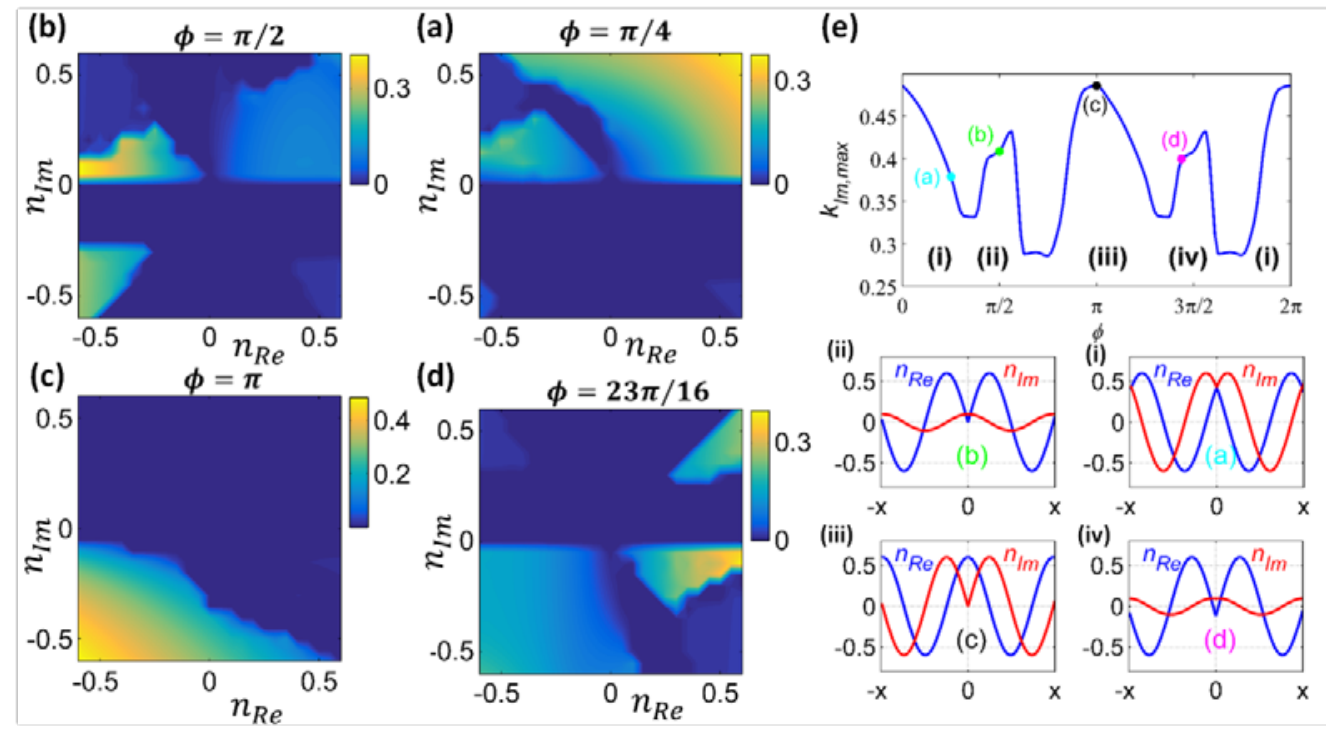

Figure 3. (a,b,c,d) Localization maps calculated after sufficient long time (tD300 units) in parameter space $\left(n_{\text {Re }}, n_{I m}\right)$ for $\phi=\pi / 4, \pi / 2, \pi$ and $23 \pi / 16$, respectively. (e) Maximum localization depending on the phase showing the strongest localization regime in different quadrants of the map: (i) $n_{R e}>0 n_{I m}>0$, (ii) $n_{R e}<0 n_{I m}>0$, (iii) $n_{R e}<0 n_{I m}<0$ and (iv) $n_{R e}>0$ $n_{I m}<0$. The index and gain/ loss potential profiles, corresponding to the phases in for the maximum localization area in (a),(b),(c), and (d) are shown in (i), (ii), (iii) and (iv), respectively, as marked on the maximum localization graph.

A direct interpretation of the different localization regimes may be based on the competition of spatial modes in the field spectra. The interaction of different growing modes gives rise to different localization regimes, depending upon the modulation amplitudes and phase.

Fourier transform does not allow a precise localization of the real components of the spectrum, as growing modes suffer from an exponential decay. The Laplace transform is an optimal method to identify the field harmonics of exponentially decaying fields. We obtain the Laplace transform numerically to analyze the most important cases. We essentially identify three distinct regimes on the 
basis of symmetric spatial modes participating in the field spectrum. The simplest case corresponds to the PhC-like limit, where a set of dominant integer modes $k_{x}=0,1,2, .$. prevail in the spatial spectrum. This is the case of points with maximal localization in Fig. 3 b with $\mathrm{n}_{\mathrm{Re}}>\mathrm{n}_{\mathrm{Im}}$ and $\phi=\pi / 2$. The other two localization regimes correspond to a GLM-like regime. In a pure GLM system, the spectrum shows frequency peaks centered at $0.5,1.5,2.5, \ldots$ and this is what we find in points with maximal localizations of Fig.3a with $\mathrm{n}_{\mathrm{Im}}>\mathrm{n}_{\mathrm{Re}}$ and $\phi=\pi / 4$. However, large gain modulation amplitudes lead to a strong coupling between different harmonics, eventually shifting the central frequency peaks towards lower and higher values, as observed in maximal localization point of Fig.3c and $\phi=\pi$ (analogous to point (c) in Fig.2b).

\section{2D AXISYMMETRIC PT-SYMMETRY}

The same combination of axial symmetry and PT-symmetry applies to the 2D-case (Fig.4). The number of possible spatial configurations of the complex index structures enlarges with dimensionality, allowing different geometries. Here, we just consider the PT-symmetric radial geometry where the coupling between incoming and outgoing waves becomes asymmetric (Fig.4a). We analyze spatial effects on beam propagation in such complex radial structures.

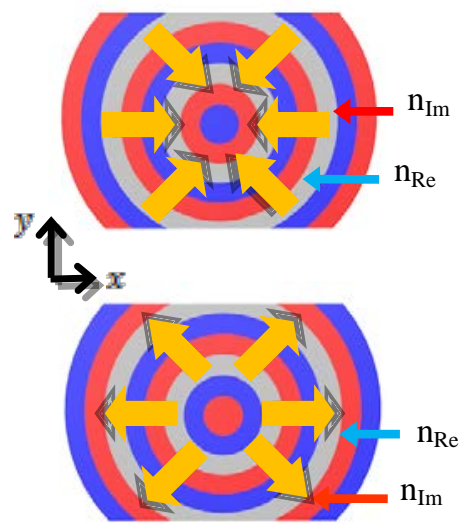

(a)

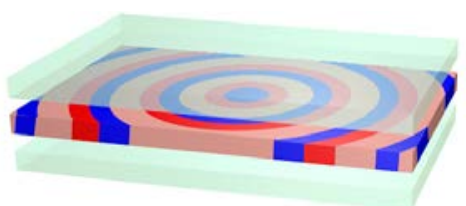

(b)

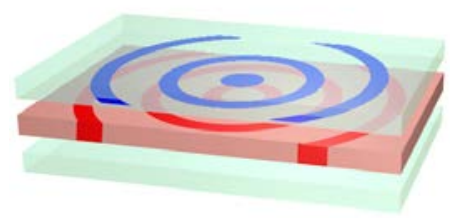

(c)

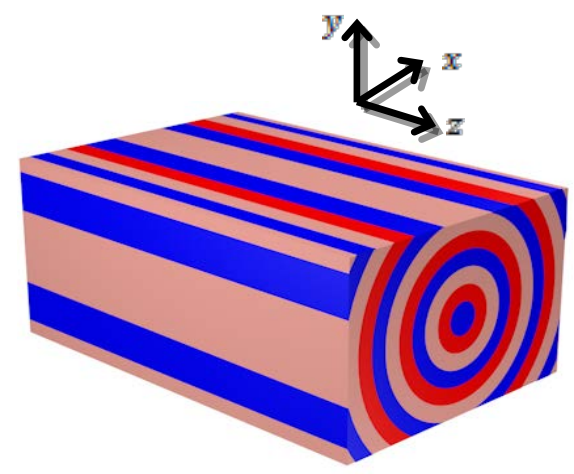

(d)

Figure 4. (a) 2D analogous axisymmetric configuration, where arrows indicate the direction of the asymmetric mode coupling. $(b, c)$ two possible realizations in broad aperture microlasers (modified VCSELS) (b) with a modulated active layer in index and gain, (c) with a gain modulated active layer and a modulated mirror. (d) 3D coaxial index and gain/loss modulations, analogous to the discussed structures for fields propagating along $\mathrm{z}$.

Different physical realizations of these arrangements 2D can be considered due to the nowadays high developed special microfabrication techniques of microchip- and VCSEL lasers. Two of the possible schemes are proposed in Fig.4b and Fig.4c for broad aperture microlasers (modified VCSELS). Moreover, the same study can be applied to the propagation of an incident beam along a 3D structure with coaxial index and gain/loss modulations just changing time by the longitudinal coordinate z corresponding to the beam propagating direction (Fig.4d).

Now, the harmonic optical refractive index represented in Fig.4 may be expressed as:

$$
n(r)=n_{\mathrm{Re}} \operatorname{Cos}(r+\phi)-n_{\mathrm{Im}} \operatorname{Sin}(r+\phi)
$$

and the light beam propagation in such structure, in analogy to the 1D case, is determined by the paraxial propagation of the electromagnetic field including diffraction and the complex potential as:

$$
\partial_{t} A(r, t)=i \nabla_{\perp}^{2} A(r, t)+i n(r) A(r, t)
$$

where $A(r, t)$ is the envelope of the field amplitude. As in the 1D case we use normalized spatial coordinates and the pair of parameters $\left(\mathrm{n}_{\mathrm{Re}}, \mathrm{n}_{\mathrm{Im}}\right)$ refers to the normalized amplitude of real and imaginary components of the complex refractive index, i.e. refractive index and gain-loss modulation amplitudes respectively. The PT-symmetry breaking points are also 
determined by $\left|\mathrm{n}_{\mathrm{Re}}\right|=\left|\mathrm{n}_{\mathrm{Im}}\right|$ and the constant phase $\phi$ characterizes the potential at the circular symmetry centre $(\mathrm{r}=0)$ and is crucial for the field localization and enhancement.

Following, we analyze the 2D PT-axisymmetric optical system characterized by eq. (2) with n(r) defined as in eq.(4). For this $2 \mathrm{D}$ case, we restrict the analysis to $\phi=0$, as it is predicted to be the optimum phase for field localization and enhancement from the 1D results; the results are summarized on Fig.5. We observe a strong field enhancement at $\mathrm{r}=0$, which may be clearly attributed to the asymmetric radial coupling between inward and outward propagation waves. The axial cross-sectional profile of the field, in logarithmic scale is not linear, due to a factor $1 / r$ of the axial symmetry. The parameter space is explored and the map of the linearized localization exponent of the axial cross section profile, obtained from $A(r) \sqrt{r}$ is shown in Fig. 5a. A high growth and extreme localization regime is found simultaneously on the top right quadrant of Fig. $5 \mathrm{a}$ and $5 \mathrm{~b}$, for $n_{\mathrm{Re}}>0 n_{\mathrm{Im}}>0$, approximately corresponding to the $1 \mathrm{D}$ results. These maps indicate that the overall scenario remains analogous to $1 \mathrm{D}$. The $2 \mathrm{D}$ field profiles in real and logarithmic scales are determined for the parameter set of point (c) in Fig. 5a, within the localization regime, and depicted in Fig. 5b(i,ii) as an example of strong field confinement around $r=0$. The general conclusion is that the situation in this $2 \mathrm{D}$ case is analogous to $1 \mathrm{D}$ with the minor advantage of larger growth exponents but with slightly smaller localization.

While the results are analogous to the simple $1 \mathrm{D}$ case, this 2D geometry is more realistic as it allows thinking in possible implementations in actual systems. In fact, as a first direct application, we expect the proposed effect to be useful to improve the emitted beam quality in broad aperture lasers.
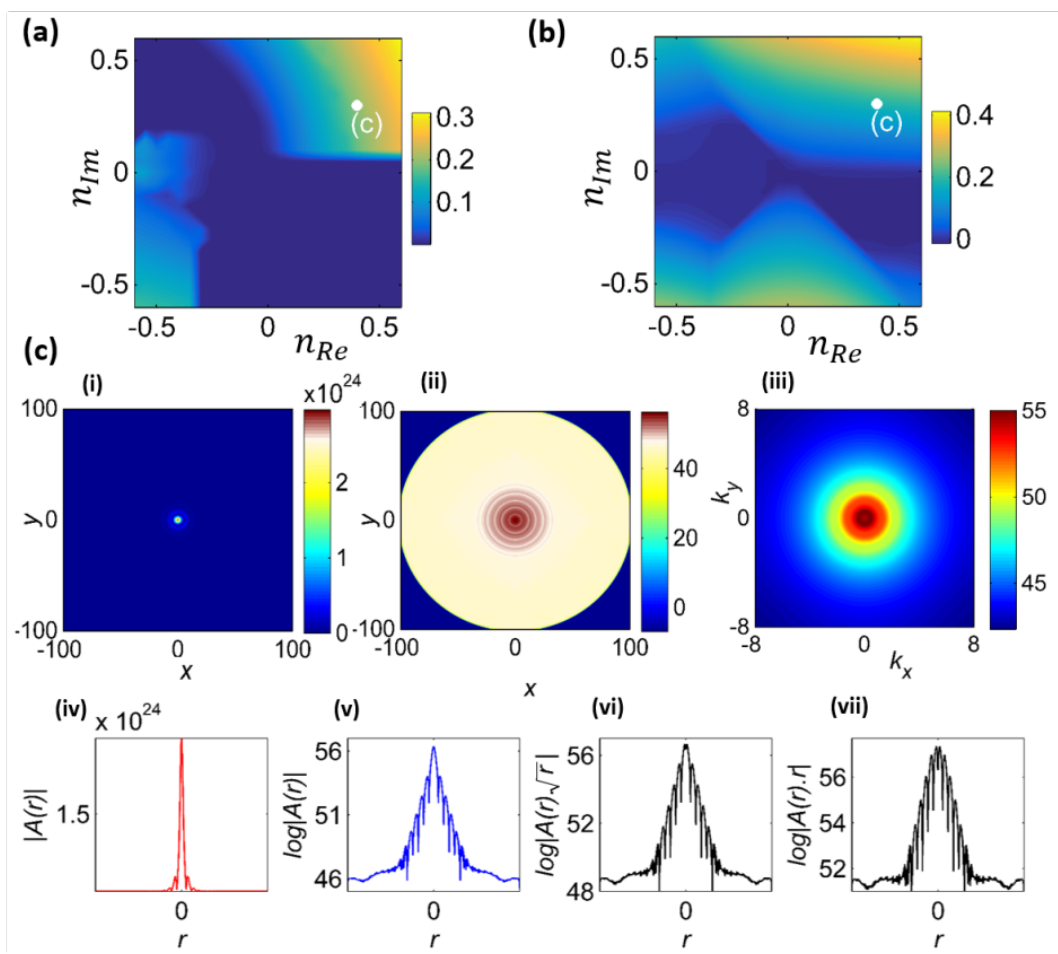

(vii)

Figure 5. (a) Localization map of the axial cross-section profile of the field $A(r) \sqrt{r}$ for $2 D$, calculated after sufficient long time (tD300 units) in parameter space $\left(n_{R e}, n_{I m}\right)$. (b) Growth map of at the center $(r=0)$ in parameter space. (c) $2 D$ spatial field profiles in real and logarithmic scales are depicted in (i), (ii) while (iii) represents the far field in logarithmic scale. The axial cross-sectional profiles of the field in real and logarithmic scales are illustrated in (iv), and (v). The localization exponents of the axial cross sectional profile, linearized as: $A(r) \sqrt{r}$ and $A(r) r$, due to the axial symmetry are shown in (vi) and (vii), respectively. 


\section{VCSELS WITH AXISYMMETRIC PT-SYMMETRY}

Broad aperture lasers, and VCSELs among them, are relevant laser sources however suffering from a major drawback a poor beam quality due to the lack of an intrinsic transverse mode selection mechanism. In simplest form, the field dynamics of VCSELS with the proposed complex potential profile satisfying the particular proposed conditions in either 1D and 2D axisymmetric conditions, may be analyzed from the nonlinear equation:

$$
\partial_{t} A(\vec{r}, t)=\left(p-|A|^{2}\right) A+i \nabla_{\perp}^{2} A(\vec{r}, t)+i n(\vec{r}) A(\vec{r}, t)
$$

where $A(r, t)$ is the envelop of complex field distributed in space, $p$ is pump parameter that defines the saturation and the complex potential profile corresponds to the one of eq.(4). Such nonlinear systems generally show the saturation phenomena with uniform field distribution for $p>0$. However, the field localization and enhancement in such situation is possible at the center for $p<0$ by applying the discussed complex refractive index. The results for a particular parameter set are provided in Fig. 6 . The spatial field profile showing the concentration at the center, $r=0$, in Figs. 6a, reveals that this 2D axisymmetric system efficiently localizes the field.

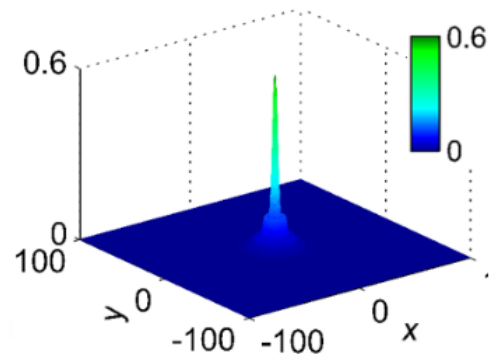

(a)

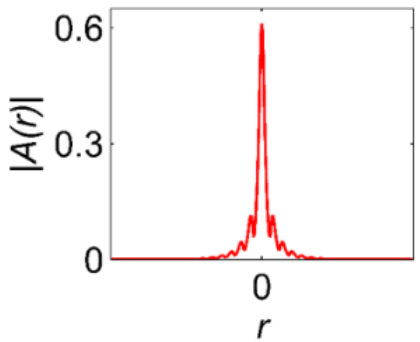

(b)

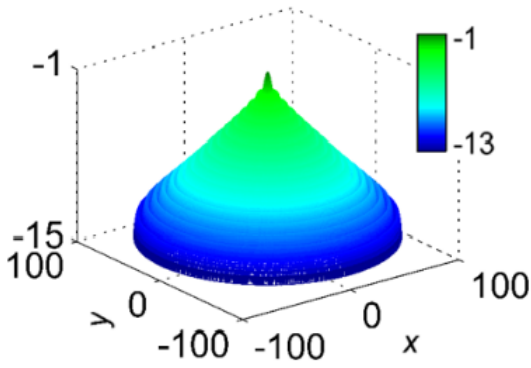

(c)

Figure 6. Spatial field distributions for a VCSEL with an axisymmetric PT-symmetry complex index, calculated after the steady state (t】150 units) for $p=-0.1$ and modulation amplitudes $\mathrm{n}_{\mathrm{Re}}=\mathrm{n}_{\mathrm{Im}}=0.4$. (a) 3D visualization of the VCSEL output field profile, concentrated and enhanced at $r=0$ in linear scale. (b) Axial cross-sectional profile. and logarithmic (b) scales. (c) 3D visualization in logarithmic scale.

\section{CONCLUSIONS}

We propose a novel class of axisymmetric $P T$-symmetric systems that provide a unique platform for strong field localization and enhancement around the central point. Such systems have the capability to simultaneously localize and enhance the field around a point due to the asymmetric coupling of inward and outward waves. We studied the parameter dependence of the localization and growth coefficients with the modulation amplitudes of refractive index and gain-loss and a phase denoting the central defect character. This light-matter interaction phenomenon may find remarkable applications in many linear and nonlinear devices where a high degree of localization is essentially desirable. One of most promising applications of the idea is to use the PT-localization property to regularize the fields in broad aperture lasers, in particular in VCSELS. Broad aperture lasers, especially the semiconductor lasers, are known to result in spatial pattern formation ${ }^{15,16}$, which usually is undesirable effect from the technological viewpoint. Different techniques to regularize the spatial structure of the emitted field are proposed, in particular the spatial modulation of the amplifying media ${ }^{17}$. The PT-like modulation proposed here could serve as advanced technique to regularize the spatial structure of output radiation. 


\section{ACKNOWLEDGEMENTS}

We acknowledge financial support by Spanish Ministerio de Ciencia e Innovación, and European Union FEDER through project FIS2015-65998-C02-01. and the Erasmus Mundus Doctorate Program Europhotonics (Grant No. 159224-12009- 1-FR-ERA MUNDUS-EMJD).

\section{REFERENCES}

[1] Staliunas, K., Herrero, R., and Vilaseca, R., "Subdiffraction and spatial filtering due to periodic spatial modulation of the gain-loss profile," Phys. Rev. A 80, 013821 (2009).

[2] Botey, M., Herrero, R., and Staliunas, K., "Light in materials with periodic gain-loss modulation on a wavelength scale," Phys. Rev. A 82, 013828 (2010).

[3] Herrero, R., Botey, M., Radziunas, M., and Staliunas, K. "Beam shaping in spatially modulated broad-area semiconductor amplifiers," Opt. Lett. 37 5253-5255 (2012).

[4] Radziunas, M., Botey, M., Herrero, R., and Staliunas, K., "Intrinsic beam shaping mechanism in spatially modulated broad area semiconductor amplifiers, "Appl. Phys. Lett. 103, 132101 (2013).

[5] Kumar, S., Herrero, R., Botey, M., Staliunas, K., "Flat lensing by periodic loss-modulated materials, " JOSA B, 30(10), 2684-2688 (2013).

[6] Kumar, S., Herrero, R., Botey, M., Staliunas, K., " Suppression of modulation instability in broad area semiconductor amplifiers, " Optics letters, 39(19), 5598-5601 (2014).

[7] Herrero, R., Botey, M., Staliunas, K., “Nondiffractive-nondiffusive beams in complex crystals”, Phys. Rev. A 89, 063811 (2014).

[8] Bender, C.M., and Boettcher, S., "Real spectra in non-Hermitian Hamiltonian having PT symmetry," Phys. Rev. Lett. 80, 5243-5246 (1998).

[9] Guo, A., et al. "Observation of PT-symmetry breaking in complex optical potentials," Phys. Rev. Lett. 103,93902 (2009).

[10] Longhi, S., "Invisibility in PT-symmetric complex crystals," J. Phys. A, Math. Theor. 44, 485302 ( 2011 ).

[11] Ruter, C.E., Makris, K. G., El-Ganainy, R., Christodoulides, D. N., Segev, M., and Kip, D., "Observation of parity-time symmetry in optics," Nat. Phys. 6, 192-195 (2010).

[12] Feng, L., Xu, Y.-L., Fegadolli, W. S., Lu, M.-H., Oliveira, J. E., Almeida, V. R., Chen, Y.-F., and Scherer, A., "Experimental demonstration of a unidirectional reflectionless parity-time metamaterial at optical frequencies," Nat. Mater. 12, 108-113 ( 2012).

[13] Lin, Z. et al. "Unidirectional invisibility induced by PT-symmetric periodic structures," Phys. Rev. Lett. 106, 213901 (2011)

[14] Regensburger, A. et al. "Parity-time synthetic photonic lattices," Nature 488, 167-171 (2012).

[15] Turduev, M., Botey, M., Giden, I., Herrero, R., Kurt, H., Ozbay, E., Staliunas, K., "Two-dimensional complex parity-time-symmetric photonic structures,“ Phy. Rev. A, 91(2), 023825. (2015).

[16] Staliunas, K., and Sanchez-Morcillo, V.J. " Transverse Patterns in Nonlinear Optical Resonators" Springer Verlag, Springer Tracts in Modern Physics, Vol.183, 2003.

[17] Tlidi, M., Staliunas, K., Panajotov, K., Vladimirov, A. G. and Clerc, M. G., "Localized structures in dissipative media: from optics to plant ecology,” Phil. Trans. R. Soc. A 372, 20140101 (2014).

[18] Ahmed, W.W., Kumar, S., Herrero, R., Botey, M., Radziunas, M., and Staliunas, K., "Stabilization of flat-mirror vertical-external-cavity surface-emitting lasers by spatiotemporal modulation of the pump profile,” Phys. Rev. A, 92, 043829 (2015). 